Method This observational survey was prospectively conducted between Jan 2010 and April 2011. Precipitants (parents or other primary care givers) of infants on home oxygen were identified through the Neonatal Outreach Referral Records. A questionnaire of 22 items was sent out to all eligible participants.

Results 18 parents/carers participated in the survey. The feedback showed that having home oxygen affected their family and social life. Support was given by health care professional regarding use of the home oxygen and safety advice in emergencies, however some of the issues identified were out of the control of the health professionals.

Conclusions Awareness of the psychological impact of discharging infants on home oxygen to the parents should be important to all health professionals involved. A standard training for the Neonatal outreach nurses and Children home Care Team should be considered in asssessment of mild depression or identifying triggers of stress prior to discharge and within the community.

\section{ALLERGY DISEASES IN CHILDHOOD AND GENERAL PEDIATRICIAN}

doi:10.1136/archdischild-2012-302724.1694

T Jakupi. Pediatric Primary Healthcare Office, Tetovo, FYR Macedonia

Aim To confirm the role of the general pediatrician in establishing the early diagnosis of allergy diseases and avoid the complications. Material and Methods Our study is prospective for the period January 2010 - December 2011. We found out that 85 children, 39 male and 46 female, from $0-18$ years old were with any kind of allergy illness. The children were divided in three groups from (0-6, 7-14, 15-15 years old). The procedures that we used to establish the diagnosis of Allergy Diseases were simple: Complete anamnesa, Questionnaires, Keeping a diary of the symptoms, Completely examination. With the help of these procedures we put initial diagnosis and confirmed the same during the skin allergy tests.

Results During this period we made skin allergy tests at 159 children. At 83 children in 2010, 76 children in 2011. From them 68 were with some allergy diseases in 2010 and 55 in 2011. With asthma were $(51 \%)$ of children, with rhinitis allergy (12\%); dermatitis allergy (13\%), vomitus and abdominal colic $2.4 \%$, laryngitis allergy $(1.2 \%)$, conjunctivitis allergy $(1.2 \%)$. While in 2011 we had $28 \%$ children with asthma, 18\% with rhinitis allergy, 18\% dermatitis allergy and urticaria, 1.3\% laryngitis allergy and 1.3\% conjunctivitis allergy. At the last we began with the adequate therapy.

Conclusion Thanks to our work we established the right diagnosis very soon, and we managed to avoid the complications which could be fatal for the child's growth.

\section{MOTHERS WITH SYSTEMIC LUPUS ERYTHEMATOSUS AND NEONATAL LUPUS. WHAT YOU NEED TO KNOW. A PRESENTATION OF 5 CASES}

doi:10.1136/archdischild-2012-302724.1695

'M Trabazo del Castillo, ${ }^{2} \mathrm{~J}$ Martínez-Baylach, ${ }^{2 B}$ Flinkenstein, ${ }^{2} \mathrm{H}$ Galdós, ${ }^{2} \mathrm{C}$ Herrera, ${ }^{1} \mathrm{G}$ Sais. 'Hospital de Mataró, ${ }^{2}$ CAP Cirera-Molins, Consorci Sanitari del Maresme, Mataró, Spain

Aim Neonatal lupus (NL) is a rare disease (1/12000-1/20000 newborns). It appears in the neonatal period of newborns of mothers with systemic lupus erythematosus (SLE) or other connective tissue diseases. It's caused by the transfer of antibodies (Anti-Ro/Anti-La) from the mother to the fetus through the placenta. We recall the main features of the NL and the management of the child who have a mother with SLE.

Methods We present 5 cases of mother-child diagnosed with SLE or NL. Four cases corresponds to mothers diagnosed with SLE (two or them, with children diagnosed of NL), and the other case is a NL with an asymptomatic mother without the diagnosis of SLE until this moment.

Results Two or the four mothers diagnosed with SLE had children with NL (one with dermatologic clinic and the other with cardiac symptoms). These two mothers presented antibodies (Anti-Ro/ Anti-La). The other two mothers didn't present these antibodies, so they had healthy children.

The fifth case was a newborn with a healthy mother, who was diagnosed with NL with dermatological features. After this, the mother was studied and had Anti-Ro/Anti-La antibodies.

No child diagnosed of NL had blood or liver disorders.

Conclusions We should study the children of mothers with SLE, particularly those with positive Anti-Ro/Anti-La antibodies, with the purpose of diagnosis of NL (particularly dermatological, cardiac, haematological and liver features).

If we suspect NL in a child of an asymptomatic mother, the presence of Anti-Ro/Anti-La antibodies in the mother and the newborn should be studies to confirm the diagnosis.

\section{SYSTEMATIC REVIEW OF ACTIVE SCHOOL TRANSPORT AND HEALTH RELATED OUTCOMES}

doi:10.1136/archdischild-2012-302724.1696

Z Yazdanpanahi, Z Molazem, S Ghadakpour. Shiraz University of Medical Sciences, Nursing and Midwifery Collage, Shiraz, Iran

Background Active commuting to school (walking or biking) may be an important opportunity for children to accumulate adequate physical activity for improved cardiovascular risk factors, enhanced bone health, and psychosocial well-being and also decreased rates of metabolic complications and cardiovascular disease in later life.

Methods This article presents the results of a systematic review of the association between active commuting to school and health related outcomes.

Results Researches shows that Childhood obesity and physical inactivity and sedentary life are increasing dramatically in worldwide. physical inactivity is closely linked to bone health, cardiovascular disease, fitness and psychological factors. Recent studies have pointed out positive effects of active commuting on physical inactivity, overweight, obesity, survivors of childhood cancer, particularly brain tumors and acute lymphoblastic leukemia also demonstrate reducing in metabolic complications and cardiovascular disease in later life.

Conclusion Physical inactivity is a risk behavior for cardiovascular and some metabolic disorder, also Active school transport (AST) may be an important and easy source of children's physical activity (PA). Schools can promote public health by educating, planning and encouraging physical activity among children.

\section{PLAYING THE GAME: WORKING WITH YOUNG CHILDREN TO DEVELOP ASTHMA INTERVENTIONS}

doi:10.1136/archdischild-2012-302724.1697

'D Bird, 'L Culley, ${ }^{3} \mathrm{M}$ Lakhanpaul, ManagementInterventions for Asthma (MIA) Research Team. ${ }^{1}$ Medical and Social Care Education, University of Leicester; ${ }^{2}$ Health and Life Sciences, De Montfort University, Leicester; ${ }^{3}$ General and Adolescent Unit, University College London, Institute of Child Health, London, UK

Background Involving children in designing healthcare interventions is encouraged but challenging and often limited to teenagers. We conducted a feasibility study assessing three methods for prioritising asthma intervention components with children aged 6-12 yrs. Methods Nineteen children prioritised 14 aspects of asthma management for intervention development using Diamond ranking (DR - equal ranks permitted), Standard Borda Ranking (SBR - no equal ranks permitted) and Budget Pie (BP - allocation of funds 